\title{
Post-Traumatic Stress Disorder and Health-Related Quality of Life in Patients and Their Significant Others Facing Lung Cancer Diagnosis: Intrusive Thoughts as Key Factors
}

\author{
Jean-Louis Pujol ${ }^{1,2}$, Carine Plassot ${ }^{1}$, Jean-Pierre Mérel $^{1}$, Elodie Arnaud ${ }^{1}$, \\ Michel Launay ${ }^{2}$, Jean-Pierre Daurès ${ }^{1}$, Isabelle Boulze ${ }^{2}$ \\ ${ }^{1}$ Thoracic Oncology Unit, Montpellier Academic Hospital, Montpellier, France \\ ${ }^{2}$ Epsylon. University Laboratory Dynamics of Human Abilities and Health Behaviours \\ EA 4556, University of Montpellier 3, Montpellier, France \\ Email: jl-pujol@chu-montpellier.fr
}

Received February 20 $0^{\text {th }}, 2013$; revised March 20 $0^{\text {th }}, 2013$; accepted April 20 $0^{\text {th }}, 2013$

\begin{abstract}
Copyright (C) 2013 Jean-Louis Pujol et al. This is an open access article distributed under the Creative Commons Attribution License, which permits unrestricted use, distribution, and reproduction in any medium, provided the original work is properly cited.
\end{abstract}

\begin{abstract}
Purpose: 1) to determine the level of post-traumatic stress disorder (PTSD) by means of impact of event scale (IES) in patients for whom lung cancer has been diagnosed for the first time and compare this level in patients having learnt that they affected by chronic obstructive pulmonary disease (COPD); 2) to compare PTSD in patients and their significant others facing a diagnosis of lung cancer; 3 ) to determine whether or not IES and General Health Questionnaire 28 (GHQ-28) correlate. Methods: This prospective survey-study included 41 consecutive patients who learnt that they were afflicted by primary lung cancer. IES and GHQ-28 were answered once between four and five weeks following medical appointment. The significant others $(\mathrm{n}=16)$ who were present during the lung cancer diagnostic appointment were also invited to complete similar questionnaires. Control group consisted of 19 patients with chronic asthma or COPD. Results: Following a lung cancer diagnosis, patients and their significant others are indistinctly affected by a high level of PTSD. These two groups had a higher frequency of high IES total score when compared with COPD patients. The IES intrusion subscale score significantly differed among groups: median [IR] values: 20 [12 - 26]; 15 [9 - 22]; 7 [5 - 15] for significant others, cancer patients and COPD patients respectively; $P=0.02$ ). IES and GHQ-28 inversely correlated. Conclusion: Patients and significant others facing a diagnosis of lung cancer are at high risk of PTSD. The level of IES intrusion in persons facing lung cancer increases emotional distress that in turn affects quality of life.
\end{abstract}

Keywords: Trauma; PTSD; Cancer Diagnosis; Lung Cancer; Significant Others

\section{Introduction}

Post-traumatic stress disorder (PTSD) is commonly observed in patients facing cancer (McBride et al., 2000; McCaul et al., 1998). Patients affected by cancer are considered to be affected by a potential traumatic event inasmuch as life-threatening illnesses are included as PTSD-leading stressor according to the DSM-IV. The first evidence classifying cancer as a stressor is that stigma and stereotypes surrounding the word "cancer" lead to the development of stressful reaction and anxiety (Themes et al., 2001; Lloyd et al., 1996). However, patients are also exposed to other stressors such as treatment consequences, major style-life changes, fears of future, family impact. The latter impact has been only partially analyzed and the detection of PTSD in patients and their relatives might be an important psychological dimension of quality of life. Among the different tools developed in order to evaluate PTSD, the impact of event scale (IES), developed by Horowitz et al. (1979) is considered as a reliable screening method. This instrument has been shown to efficiently measure avoidance and intrusion cognitions following any traumatic event. In the past, several studies have used
IES in order to evaluate PTSD incidence among different populations of cancer patients (Jacobsen \& Holland, 1991; Norum \& Wist, 1996; Kaasa et al., 1993).

Lung cancer is known to rapidly impair quality of life (Gralla $\&$ Thatcher, 2004), and usually requires intensive therapy resulting in the daily involvement of significant others with regards to everyday care and living with the disease (Ostlund et al., 2010). The distress that affects significant others, particularly spouses, is probably underestimated (Gaugler et al., 2008). The diagnosis of lung cancer is a highly traumatic event, given the social presuppositions of both incurable and self-inflicted disease that are conveyed by this diagnosis (Chapple et al., 2004) and therefore may differ from other cancers (LoConte et al., 2008; Pujol \& Quantin, 2009). We hypothesized that both patients and significant others are at high risk of PTSD following a lung cancer diagnosis.

The herein study was designed in order 1) to determine the level of PTSD by means of impact of event scale (IES) in patients for whom lung cancer has been diagnosed for the first time and compare this level in patients having learnt that they affected by (chronic obstructive pulmonary disease (COPD); 2) 
to compare PTSD in patients and their significant others facing a diagnosis of lung cancer; 3 ) to determine whether or not there is any correlation between IES and General Health Questionnaire 28 (GHQ-28, QoL scale measuring emotional distress in medical settings).

\section{Participants and Methods}

\section{Study Design}

This is a prospective survey-study. The survey was designed to take 20 minutes to complete, and included validated tools, demographic data and disease characteristics. Lung cancer patients and their significant others were consecutive participants seen during a chest medical oncology consultation with one of our team members. During this consultation, the lung cancer was diagnosed to the patient in presence of his/her relative. Then, a delay of at least four weeks (and no more than 5 weeks) elapsed before the different questionnaires were administered. During the period of this survey-study, patients with chronic respiratory symptoms who attended a preplanned consultation with the same physician in the outpatient unit were invited to participate once the diagnosis of their benign obstructive lung disease (asthma or COPD) had been established. The questionnaires were collected by an administrative representative who was responsible for keeping the records anonymous and who managed a clinical research technical assistant, the latter responsible for recording the data. The database was frozen on March 5th, 2012. The questionnaires were completed by the patients either in a quiet room of the unit or at home for those preferring not to answer immediately. The questionnaires were answered once between four and five weeks following medical appointment. None of the patients were asked to answer in presence of a physician, nurse or psychologist. All participants spoke and wrote French fluently. Written informed consent indicating the purpose of the research and guarantying the anonymous evaluation of their answers was given by all participants. The study was approved by the Montpellier Ethical Committee.

\section{Patients}

Consecutive patients of both sexes, aged over 18 and who were seen for the first time at the Montpellier Academic Hospital chest oncology consultation unit were prospectively accrued on the basis that lung cancer diagnosis was announced for the first time during this first consultation. The eligibility criteria consisted of: 1) histologically- or cytologically-proven lung cancer of any stage (usually incompletely known at that time) and any histology (both small cell and non-small cell lung cancer), 2) performance status equal to or less than 2 according to the Eastern Cooperative Oncology Group (ECOG), 3) no previous history of malignant disease (except well-controlled basal cell skin cancer or in situ cervix carcinoma).

\section{Significant Others}

Those who took part as "significant others" during the diagnostic consultation were invited to participate in the study, whatever the degree of relation (generally the patient's spouse but occasionally a parent, adult child or sibling). The eligibility criteria consisted of being aged 18 years or above.

\section{Control Group}

Consecutive patients suffering from chronic or recurrent respiratory symptoms, who learnt that they were afflicted with a diagnosis of disabling chronic asthma or COPD entered the study as part of the control group. Diagnoses were established according to current international guidelines and took into account duration/characteristics of respiratory symptoms prior to visit in the unit, and results of respiratory functions tests performed in the outpatient unit of the chest department of the Montpellier Academic Hospital. The eligibility criteria consisted of the following: 1) aged 18 years or above, 2) suffering from chronic asthma with a Global Initiative for Asthma (GINA) score of $3-4$ or COPD with a Chronic Obstructive Lung Disease (GOLD) score $\geq 2,3$ ) symptoms of dyspnoea or wheezing, interfering with activity and requiring daily treatment, 4) no previous history of cancer at any site.

\section{Questionnaires}

Putative predictive variables to be tested: The participants' demography and social position questionnaire consisted of the following items: sex, age, marital status (single, divorced, married), smoking habits (never smoker, former smoker [at least one year since stopping], active smoker), employment, educational degree, knowledge of cancer (having been affected him/ herself, being a relative of an affected person or having been informed by the general media).

Impact of event scale: The IES has been elaborated to assess psychological stress reactions after any major life event and has been validated in any kind of stressful event including cancer over the past three decades (Horowitz et al., 1979; Koopman et al., 2004; Steinglass \& Gerrity, 1990; Tibben et al., 1994). In this study, it has been anchored to the date of the event: i.e. the date of the cancer diagnosis for the lung cancer patients and their significant others; the date of diagnosis of COPD or chronic asthma for the control group. The IES consists of 15 items that measure intrusive re-experiences of the trauma (seven questions) and avoidance of trauma-related stimuli (eight questions). Participants are invited by each of the 15 questions to report the frequency of symptoms during the past seven days on a four-point scale: 0 indicating not at all, 1 indicating rarely, 3 indicating sometimes, and 5 indicating often. By combining the 15 items, the IES total score, ranging from 0 to 75 , can be calculated for each participant. The IES enables a separate analysis of intrusion and avoidance subscale scores (with a possible range of $0-35$ and $0-40$, respectively). The original IES questionnaire was used in its validated French translated form.

Global Health Questionnaire 28: The GHQ-28 is a measure of generalized psychological distress and has been validated for a wide range of populations and a variety of settings (Goldberg $\&$ Hillier, 1979). This 28 -item health-related quality of life instrument is mainly devoted to measure emotional distress in medical settings. A score of above 10 indicates that the psychological distress level is consistent with a need for psychological intervention. The GHQ comprises 28 items, seven of which are formulated in a positive manner (e.g. Do you feel perfectly well and in good health?) and 21 in a negative manner (e.g. Do you feel sick?). The four subscales of the GHQ-28 are severe depression, social dysfunction, anxiety and insomnia, and somatization. These were calculated separately and then pooled in the total GHQ-28 score. The original GHQ-28 questionnaire was used in its validated French translated form (Bo- 
lognini et al., 1989).

\section{Statistics}

Comparisons between groups were carried out as follows: the distribution of qualitative variables (such as gender, employment, educational degree, etc) between groups was compared using the $\chi^{2}$ test. When the calculated frequency of the categorical data of the contingency table did not allow the use of the $\chi^{2}$ test, the Fisher's exact test was used. The normal (Gaussian) distributions of the IES scores and GHQ-28 scores were tested using the non-parametric Kolmogorov-Smirnov test (K-S test) for the equality of continuous, one-dimensional probability distribution: Regarding the GHQ-28 total scores, in each of the three tested populations, the $P$ value was $>0.10$, indicating a normal distribution. Therefore, the expression of GHQ-28 scores as mean \pm S.D. was allowed. Regarding IES total and subscale scores, the tests were significant, thereby rejecting the $\mathrm{H} 0$ hypothesis (i.e. demonstrating that IES scores were not distributed according to the Gaussian law). Therefore, in order to analyze the distribution of variables such as IES scores among groups, results were expressed as median, and variation was expressed as interquartile range [IR]. Non-parametric statistical analyses were uniformly used: differences between two independent groups were determined by means of the Mann Whitney U test; differences between more than two groups were determined by the Kruskal Wallis one-way analysis of variance.

In order to determine the sensitivity-specificity relationship of IES total score, IES intrusion structure score and IES avoidance score in detecting PTSD following lung cancer diagnosis, receiver Operating Characteristic (ROC) curves were constructed: sensitivity was considered as a true positive (i.e. high IES score or subscores) in lung cancer patients and specificity was considered as a true negative (i.e. low IES score or subscores) in patients suffering from chronic asthma or COPD. Areas under the ROC curves (AUC-ROC) were calculated. The $\mathrm{Z}$ statistic (two-tailed test) was applied for comparing each AUC-ROC and non-information line. Correlations between total GHQ-28 and IES were made as follows: Separate scatterplots of IES total score, IES intrusion score and IES avoidance score, by GHQ-28 score, were tested using Spearman rankorder correlation coefficients. Internal consistency was tested across subscales and for the total IES in all participants: item total correlation coefficients and Chronbach's $\alpha$ coefficient for the intrusion and avoidance subscales of the IES were calculated. A $P$ level of less than 0.05 was considered as significant. SAS software was used for all analyses (version 9.2, Copyright (c) 2002-2008 by SAS Institute Inc., Cary, NC, USA).

\section{Results}

The herein reported results suggest that patients facing a lung cancer diagnosis together with their significant others have been affected by a high level of intrusive thoughts.

\section{Description of Samples}

Between July 2011 and March 2012, a total of 95 participants were invited to take part in this survey-study (Table 1). Among them, 19 (20\%) withdrew their agreement to participate (five in the control group, ten in the patients group and four in the significant others group). The herein study describes the
Table 1.

Participants' demographic and social positioning.

\begin{tabular}{|c|c|c|c|c|}
\hline & $\begin{array}{l}\text { Lung cancer } \\
\text { pts } \mathrm{n}(\%)\end{array}$ & $\begin{array}{c}\text { Relatives } \\
\text { n (\%) }\end{array}$ & $\begin{array}{l}\text { COPD } \\
\mathrm{n}(\%)\end{array}$ & $P^{\dagger}$ \\
\hline $\mathrm{N}$ & 41 & 16 & 19 & \\
\hline Age (median) & 59 & 60.5 & 57 & 0.54 \\
\hline Women & $18(43.90)$ & $12(75.00)$ & $9(47.37)$ & 0.69 \\
\hline Marital status & & & & 0.39 \\
\hline Single & $4(9.76)$ & & $3(15.79)$ & \\
\hline Divorced & $6(14.63)$ & & $3(15.79)$ & \\
\hline Married & $31(75.61)$ & $16(100)$ & $13(68.42)$ & \\
\hline Employment & & & & 0.39 \\
\hline Working & $15(36.59)$ & $5(31.25)$ & $9(47.37)$ & \\
\hline Retired & $18(43.90)$ & $8(50.00)$ & $10(52.63)$ & \\
\hline Unemployed & $7(17.07)$ & $3(18.75)$ & & \\
\hline Missing data & $1(2.44)$ & & & \\
\hline Knowledge of cancer & & & & 0.004 \\
\hline Personal experience & $31(75.61)$ & $1(6.25)$ & $1(5.26)^{*}$ & \\
\hline Relative affected & $8(19.51)$ & $13(81.25)$ & $11(57.89)$ & \\
\hline None of the above & $2(4.88)$ & $2(12.50)$ & $5(26.32)$ & \\
\hline Smoking habits & & & & 0.004 \\
\hline Never smoker & $3(7.32)$ & $6(37.50)$ & $10(52.63)$ & \\
\hline Former smoker & $30(73.17)$ & $5(31.25)$ & $8(42.11)$ & \\
\hline Active smoker & $8(19.51)$ & $5(31.25)$ & $1(5.26)$ & \\
\hline Educational degree & & & & 0.18 \\
\hline A level or less & $24(58.54)$ & $6(37.50)$ & $8(42.11)$ & \\
\hline Bachelor or higher & $16(39.02)$ & $10(62.50)$ & $11(57.89)$ & \\
\hline Missing data & $1(2.44)$ & & & \\
\hline
\end{tabular}

Statistical comparison between the three groups: Mann and Whitney $U$ test for age; comparisons using $\chi^{2}$ tests for all others; " protocol violation.

survey of the remaining 76 participants: 41 lung cancer patients, 16 significant others and $19 \mathrm{COPD} /$ chronic asthmatic patients. Knowledge of cancer from the participants' point of view had different origins depending on the groups and as a consequence of the group definitions themselves (e.g. most of the participants in the significant others group had a knowledge of cancer from being the spouse of a patient with lung cancer). Smoking habits significantly differed within the groups. Other variables tested did not significantly differ among groups. In particular, subscales of the GHQ-28 detected a comparable level of these dimensions among patients with lung cancer and patients with COPD, suggesting that groups did not differ in terms of burden of symptoms. There were five missing items among the 76 IES questionnaires (0.04\% missing data) and four missing subscales among the 76 GHQ-28 questionnaires (1.32\% missing data). 


\section{Sensitivity-Specificity Relationship of IES}

Specificity was calculated using the results of the IES scores in the control group, and sensitivity was measured in the patients with lung cancer. AUC-ROC curves [95\% confidence interval] and $Z$ statistics were 0.65 [0.52 - 0.79], $P=0.04 ; 0.69$ [0.56 - 0.81], $P=0.01$; and 0.60 [0.46 - 0.74], $P=0.18$ for the IES total score, IES intrusion subscale score and IES avoidance subscale score respectively (Figure 1). Therefore, the IES total score and intrusion subscale score AUC-ROC curves significantly differed from the non-information line, whereas the IES avoidance subscale score did not. The IES total score threshold of 35 was used throughout the following steps of this study; this threshold is characterizing stress-response to major traumatizing events as published in the literature (Sundin \& Horowitz, 2003).

\section{IES Distribution According to Groups}

Taking into account the threshold, the patients with lung cancer and the cancer-patient significant others had a higher frequency of high IES total score when compared with patients with COPD or chronic asthma (49\% versus $21 \% ; \chi^{2}$ test, $P=$ 0.04; 1 degree of freedom).

The IES intrusion subscale score significantly differed among groups inasmuch as the highest median [IR] values were observed in the significant others group and the lowest in the control group, (20 [12 - 26]; 15 [9 - 22]; 7 [5 - 15] for significant others, patients with lung cancer and control group respectively; Kruskal Wallis, $P=0.02$; Figure 2(a)). The IES intrusion subscale score was significantly lower in the latter group when compared with the patients with lung cancer group (Mann and Whitney $U$ test, $P=0.04$ ) and the significant others group (Mann and Whitney $U$ test, $P=0.01$ ). The IES intrusion subscale score in the significant others group was higher than that observed in the patients with lung cancer group, although the difference did not reach the level of statistical significance (Mann and Whitney U test, $P=0.14$ ).

The IES avoidance subscale score did not significantly differ among the groups, with median [IR] values of 9 [4.5 - 15]; 14 [7 - 22]; 8 [3 - 18] for significant others, patients with lung cancer and control group respectively (Kruskal Wallis, $P=0.17$ Figure 2(b)).

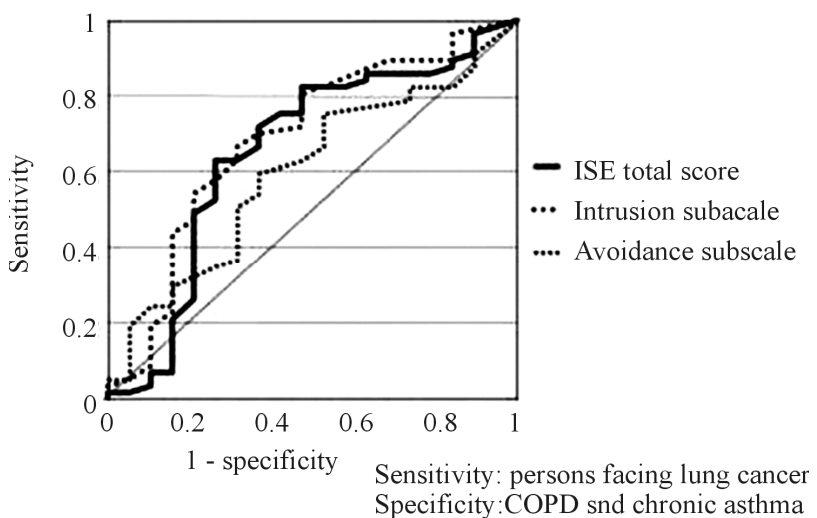

Figure 1.

Receiver operating characteristic constructed using the sensitivityspecificity relationship of IES scale and subscales to discriminate $\mathrm{COPD} /$ chronic asthmatic patients and persons facing lung cancer.

\section{GHQ-28 and IES Relationship}

The GHQ-28 total score did not significantly differ among the groups with mean \pm SD: $8.13 \pm 4.94,7.59 \pm 5.18$ and $7.05 \pm$ 5.03 for significant others, patients with lung cancer and control group respectively (Kruskal Wallis, $P=0.77$ ). The four subscales of the GHQ-28 did not significantly vary among the different groups (data not shown). There was a significant relationship of IES total score and GHQ-28 total score with a Spearman rank-order correlation coefficient $\mathrm{Rs}=0.51(P<$ 0.0001 ; Figure 3).

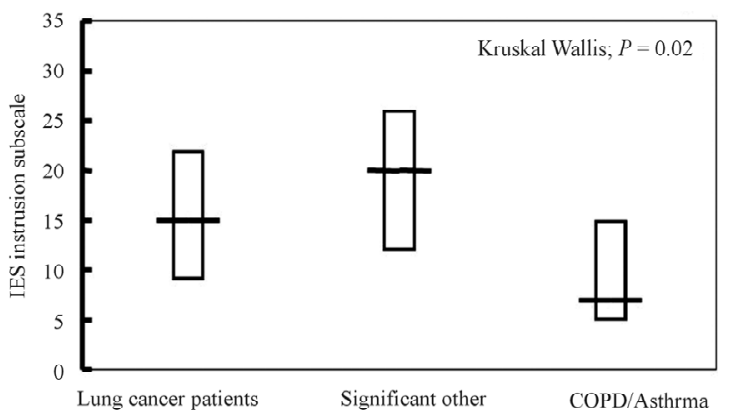

(a)

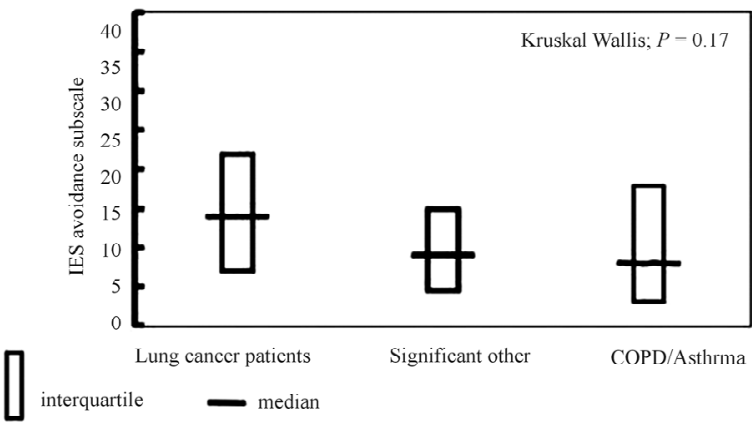

(b)

Figure 2.

Impact of event scale score distribution according to groups. Horizontal $\mathrm{bar}=$ median value; columns $=$ interquartile range. (a) Intrusion subscale (Kruskal-Wallis Test: $P=0.02$ ); (b) Avoidance subscale (Kruskal-Wallis Test: $P=0.17$ ).

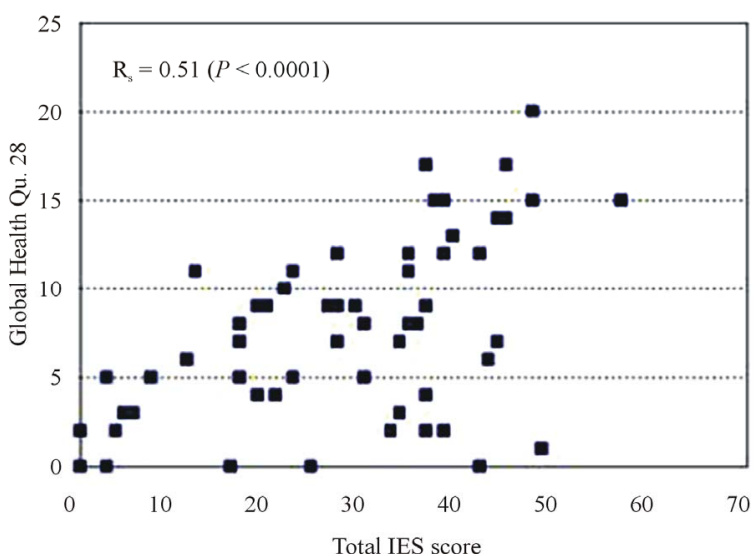

Figure 3.

Relationship of IES total score and GHQ-28 total score in persons facing lung cancer. Spearman rank-order correlation coefficient Rs $=0.51(P<0.0001)$. 
The distribution of IES did not significantly vary according to the age, family status, smoking habit, employment, degree of education. However, women proved to have a higher IES total score when compared with men (median [IR]: 36.5 [29 - 45] versus 22 [12 - 39]; Mann and Whitney U test: $=0.03$ ).

\section{Internal Consistency}

In Table 2, Cronbach's $\alpha$ and item total correlation coefficients are shown. Internal consistency coefficients proved to be uniformly high across subscales and for the total IES in the herein whole population. Cronbach's $\alpha$ were $0.88,0.90$ and 0.81 for IES total score, intrusion subscale and avoidance subscale respectively. Each subscale was found to be homogeneous with satisfactory correlations between each item and subscale total scores.

\section{Discussion}

Three different data sets emerging from this survey-study would merit discussion as they might be of importance to clinicians proposing best therapy and psychological support to lung cancer patients: 1) Post-traumatic stress disorders following the diagnosis of lung cancer are extremely high, 2) the significant other is consistently affected, having co-experienced the same

Table 2.

Item total correlation coefficients and Cronbach's $\alpha$ coefficients for the intrusion and avoidance subscales of the IES participants' demographic and social positioning.

\begin{tabular}{|c|c|c|}
\hline $\begin{array}{c}\text { Item } \\
\text { intrusion items }\end{array}$ & Item-total correlation coefficient & Cronbach's $\alpha$ \\
\hline 1 & 0.71 & 0.88 \\
\hline 4 & 0.68 & 0.88 \\
\hline 5 & 0.78 & 0.87 \\
\hline 6 & 0.57 & 0.89 \\
\hline 10 & 0.81 & 0.87 \\
\hline 11 & 0.62 & 0.89 \\
\hline \multirow[t]{2}{*}{14} & 0.72 & 0.88 \\
\hline & Subscale total Cronbach's $\alpha=0.90$ & \\
\hline \multicolumn{3}{|l|}{ Avoidance items } \\
\hline 2 & 0.40 & 0.81 \\
\hline 3 & 0.58 & 0.78 \\
\hline 7 & 0.46 & 0.80 \\
\hline 8 & 0.34 & 0.82 \\
\hline 9 & 0.55 & 0.79 \\
\hline 12 & 0.71 & 0.77 \\
\hline 13 & 0.68 & 0.77 \\
\hline \multirow[t]{4}{*}{15} & 0.52 & 0.79 \\
\hline & Subscale total Cronbach's $\alpha=0.81$ & \\
\hline & Total IES scale & \\
\hline & Total score Cronbach's $\alpha=0.88$ & \\
\hline
\end{tabular}

trauma, 3) intrusive re-experiences are the main stress reactions and might explain the high values of observed IES.

In the herein study, an IES value greater than or equal to 35 is very common and affects most of the patients with lung cancer following the event (diagnosis). This is in accordance with several studies that have noted that patients with lung cancer are affected by a much greater stigma than patients with cancer emerging in other sites (Bell et al., 2010; Burris, 2006). This is due to the widespread social representations that constantly consider lung cancer as a self-inflicted disease, even if this social thinking is disconnected from scientific reality. Indeed, over the last two decades, there has been an epidemiological rise of lung cancer among non-smoker (or light former smoker) patients. According to qualitative psychological studies, nonsmoker patients suffer from similar stereotypes and stigmas and must constantly defend themselves as having no responsibility in their disease (Chapple et al., 2004).

In an article by LoConte et al. (2008), a group of patients with non-small cell lung cancer (NSCLC) was compared to a group of patients with either breast cancer or prostate cancer. This study is interesting because the social representations of these diseases are quite distinct. Patients with prostate cancer, like patients with breast cancer, are not generally considered responsible for their illness. Moreover, the social representation of these diseases does not systematically link them with a poor prognosis. These authors have shown that NSCLC patients had high levels of perceived stigma related to cancer and that they were much higher than those of patients with prostate or breast cancer. Their smoking status might have significantly correlated with high levels of guilt and shame (Bell et al., 2010). In a classic paper, Sundin and Horowitz (2003) performed a metaanalysis of the use of IES during two decades. They demonstrated that it was possible to rank the events by type, according to the mean IES levels observed in different studies: the group of subjects facing an illness or injury is usually part of the lower hierarchical levels and this could be the case in our study of patients with COPD or chronic asthma.

The fact that the diagnosis of COPD, a disease with a severely disabling health condition, induced a PTSD with IES scores that ranked in the group of diseases or injury according to the meta-analysis by Horowitz is congruent. A lot more surprising is the fact that the diagnosis of lung cancer is, for the person who receives this diagnosis, a traumatic event whose consequences in terms of post-traumatic stress are within the values reported by Horowitz that affect survivors of scenes of war (Sundin \& Horowitz, 2003). The reality of post-traumatic stress disorder is evidenced by the internal consistency coefficients of all of the items of the IES scale and subscales together with the correlation of the IES scores and quality of life GHQ28 scores in persons facing lung cancer (Haagsma et al., 2012).

An increased amount of literature suggests that the psychological distress of the significant others interacting with the person suffering from cancer could have been underestimated (Ostlund et al., 2010). In this setting, there are specificities regarding significant others, and here again they are in link with the unique characteristics of the lung cancer itself. Significant others are as exposed to the representations of this illness as are the patients themselves (the characteristics included in this representation, such as almost incurable disease and/or self-inflicted disease, are generalized in occidental countries). Another feature is due to the high level of symptoms. Gralla (2012) 
noted that over $80 \%$ of patients with lung cancer are affected by at least three symptoms (including the most common such as pain, dyspnea and fatigue). This explains the patient's social function impairment. Therapeutic weight by itself also contributes to this impairment and consequently increases the impact in the significant other's life, with possible changes in family and social status. Caught between the social representations of illness and the burden of patient support, the significant other's room for expression is restricted during or subsequent to the diagnosis.

From the significant other's point of view, the herein study suggests that the trauma is equivalent to that affecting the patients, as long as the IES total scores and IES intrusion subscale scores are considered. Here again, internal consistency coefficients of all items of the IES scale and subscales together with the correlation of the IES scores and quality of life GHQ-28 scores attest the reality of the observation (Ganz et al., 1991; Dancey et al., 1997). Therefore, this study suggests that the diagnosis-induced trauma had as much impact (and maybe numerically more) on the significant other's experience as on the patient's, and that the GHQ-28 is essentially a health-related quality of life instrument. Considering the intrusion subscale score, the high median value in asymptomatic significant others and the low median value in COPD patients (although suffering from symptoms requiring daily treatment) demonstrated that there was no link between clinical burden and a high IES.

The intrusive re-experiences are more important than the avoidance cognitions after the specific event of lung cancer diagnosis. We hypothesize that avoidance type behaviors are inhibited because of the current circumstances of diagnosis and treatment of lung cancer. Indeed, the majority of patients who participated in this survey-study were then supported by therapy, mainly with chemotherapy regimens given every three weeks. The repetitive admissions did not allow favorable conditions for avoidance cognitions. As a matter of fact, several studies having evaluated PTSD prevalence in various malignant diseases, suggested that avoidance behavior is difficult to define in cancer patients insofar as therapy imply a continuous confrontation with putative stressors (Mehnert \& Koch, 2007). Intrusive re-experiences are high (some patients' questionnaires having indicated the 35 maximum subscale score); this could be interpreted as an indicator of a particularly intense break at the time of the diagnosis. This study has two main limitations: the follow up is not sufficiently long to assess possible change along time of the PTSD level. Comparison with other cancers that are not linked with self-inflicted social representations should be useful. However, the herein reported results deserve further researches.

Psychological intervention should be considered in any patient with an IES score equal to or greater than 35 and/or a high level of intrusive re-experiences. A similar intervention should also be offered to significant others as they experience the same type of PTSD.

\section{Acknowledgements}

Authors thank Mrs. Sylvia Motsch and Mrs. Odile Flohic for technical assistance. Pr Gregory Ninot; Pr Claude Guy Bruère Dawson for helpful discussions. Study supported by grants from the French League against Cancer (national and Hérault committee), from Roche Company and from Chugaï Company.

\section{REFERENCES}

Bell, K., Salmon, A., Bowers, M., Bell, J., \& McCullough, L. (2010). Smoking, stigma and tobacco "denormalization": Further reflections on the use of stigma as a public health tool. Social Science \& Medicine's Stigma, Prejudice, Discrimination and Health, 70, 795-799. doi:10.1016/j.socscimed.2009.09.060

Bolognini, M., Bettschart, W., Zehndergubler, M., \& Rossier, L. (1989). The validity of the french version of the GHQ-28 and psydis in a community sample of 20 years olds in Switzerland. European Archives of Psychiatry and Neurological Sciences, 238, 161-168. doi:10.1007/BF00451005

Burris, S. (2006). Stigma and the law. Lancet, 367, 529-531. doi:10.1016/S0140-6736(06)68185-3

Chapple, A., Ziebland, S., \& McPherson, A. (2004). Stigma, shame, and blame experienced by patients with lung cancer: Qualitative study. British Medical Journal, 328, 1470.

doi:10.1136/bmj.38111.639734.7C

Dancey, J., Zee, B., \& Osoba, D. (1997). Quality of life scores: An independent prognostic variable in a general population of cancer patients receiving chemotherapy. The National Cancer Institute of Canada Clinical Trials Group. Quality of Life Research, 6, 151-158. doi:10.1023/A:1026442201191

Ganz, P. A., Lee, J. J., \& Siau, J. (1991). Quality of life assessment. An independent prognostic variable for survival in lung cancer. Cancer, 67, 3131-3135.

doi:10.1002/1097-0142(19910615)67:12<3131::AID-CNCR2820671 $232>3.0 . \mathrm{CO} ; 2-4$

Gaugler, J. E., Given, W. C., Linder, J., Kararia, R., Tucker, G., \& Regine, W. F. (2008). Work gender and stress in family cancer caregiving. Support Care Cancer, 16, 347-357. doi:10.1007/s00520-007-0331-y

Goldberg, D. P., \& Hillier, V. F. (1979). A scale version of the General Health Questionnaire. Psychological Medicine, 9, 139-145. doi:10.1017/S0033291700021644

Gralla, R. J., \& Thatcher, N. (2004). Quality-of-life assessment in advanced lung cancer: Considerations for evaluation in patients receiving chemotherapy. Lung Cancer, 46, S41-S47. doi: $10.1016 / \mathrm{S} 0169-5002(04) 80040-0$

Gralla, R. J. (2012). Coming of age for monitoring quality of life and patient-reported outcomes. Journal of Thoracic Oncology, 7, 8-9. doi:10.1097/JTO.0b013e31823f12a3

Haagsma, J. A., Polinder, S., Olff, M., Toet, H., Bonsel, G. J., \& Van Beeck, E. F. (2012). Postraumatic stress symptoms and health-related quality of life: A two year follow up study of injury treated at the mergency department. BMC Psychiatry, 12, 1. doi:10.1186/1471-244X-12-1

Horowitz, M. J., Wilner, N. R., \& Alvarez, W. (1979). Impact of event scale: A measure of subjective stress. Psychosomatic Medicine, 41, 209-218.

Jacobsen, P. B., \& Holland, J. C. (1991). The stress of cancer: Psychological responses to diagnosis and treatment. In: C. I. Cooper, \& M. Watson (Eds.), Cancer and stress: Psychological, biological and coping studies (pp. 147-169). Chichester: Wiley.

Kaasa, S., Malt, U., Hagen, S., Wist, E., Moum, T., \& Kvikstad, A. (1993). Psychological distress in cancer patients with advanced disease. Radiotherapy \& Oncology, 27, 193-197. doi:10.1016/0167-8140(93)90073-H

Koopman, C., Classen, C., \& Spiegel, D. (1994). Predictors of posttraumatic stress symptoms among survivors of the Oakland/Berkeley, Calif., firestorm. American Journal of Psychiatry, 151, 888-894.

Lloyd, S., Watson, M., Waites, B., Meyer, L., Eeles, R., Ebbs, S., \& Tylee, A. (1996). Familial breast cancer: A controlled study of risk perception, psychological morbidity and health beliefs in women attending for genetic counseling. British Journal of Cancer, 74, 482487. doi:10.1038/bjc. 1996.387

LoConte, N. K., Else-Quest, N. M., Eickhoff, J., Hyde, J., \& Schiller, J. H. (2008). Assessment of guilt and shame in patients with non-smallcell lung cancer compared with patients with breast and prostate cancer. Clinical Lung Cancer, 9, 171-178.

doi:10.3816/CLC.2008.n.026 
McBride, C. M., Clipp, E., Peterson, B. L., Lipkus, I. M., \& DemarkWahnefried, W. (2000). Psychological impact of diagnosis and risk reduction among cancer survivors. Psycho-Oncology, 9, 418-427. doi:10.1002/1099-1611(200009/10)9:5<418::AID-PON474>3.0.CO; 2-E

McCaul, K. D., Branstetter, A. D., O'Donnell, S. M., Jacobson, K., \& Quinlan, K. B. (1998). A descriptive study of breast cancer worry. Journal of Behavioral Medicine, 6, 565-557. doi: $10.1023 / \mathrm{A}: 1018748712987$

Mehnert, A., \& Koch, U. (2007). Prevalence of acute and post-traumatic stress disorder and comorbid mental disorders in breast cancer patients during primary cancer care: A prospective study. Psychooncology, 16, 181-188. doi:10.1002/pon.1057

Norum, J., \& Wist, E. (1996). Psychological distress in survivors of Hodgkin'disease. Support Care Cancer, 4, 191-195.

doi:10.1007/BF01682339

Ostlund, U., Wennman-Larsen, A., Persson, C., Gustavsson, P., \& Wengstrom, Y. (2010). Mental health in significant others of patients dying from lung cancer. Psycho-Oncology, 19, 29-37. doi:10.1002/pon.1433
Pujol, J. L., \& Quantin, X. (2009). Time to diagnosis of lung cancer: Technical and pyschological factors that slow down diagnostic and treatment timelines. Journal of Thoracic Oncology, 4, 1192-1194. doi:10.1097/JTO.0b013e3181b88045

Steinglass, P., \& Gerrity, E. (1990). Natural disasters and post-traumatic stress disorder: Short-term versus long-term recovery in two disaster-affected communities. Journal of Applied Social Psychology, 20, 1746-1765. doi:10.1111/j.1559-1816.1990.tb01509.x

Sundin, E. C., \& Horowitz, M. J. (2003). Horowitz's impact of event scale evaluation of 20 years of use. Psychosomatic Medicine, 65, 870-876. doi:10.1097/01.PSY.0000084835.46074.F0

Themes, B., Meiser, B., \& Hickie, I. B. (2001). Psychomemtric properties of the impact of event scale amongst women at increased risk for hereditary breast cancer. Psycho-Oncology, 10, 459-468. doi: $10.1002 /$ pon. 533

Tibben, A., Duivenvoorden, H. J., Niermeier, M. F., Vegter-Van Der Vlis, M., Roos, R. A. C., \& Verhage, F. (1994). Psychological effects of presymptomatic DNA testing for Huntington's disease in a Dutch program. Psychosomtric Medicine, 56, 526-532. 